CASE REPORT

\title{
Use of Beta-D-Glucan for Monitoring Antifungal Therapy of a Multidrug Resistant Invasive Magnusiomyces capitatus Infection
}

\author{
Shrijit Nair, Rosa Miquel, Wayel Jassem, Abid Suddle, Anita Verma \\ King's College Hospital, Denmark Hill, London, United Kingdom
}

\begin{abstract}
Invasive fungal infection (IFI) with Magnusiomyces capitatus is rare, occurring mainly in patients with hematological diseases. We report first case of tissue invasive $M$. capitatus infection in a liver transplant recipient (LTR) and use of an IFI biomarker beta-D glucan (BDG) for monitoring antifungal therapy.

A 59 year old male received 3rd liver transplant (LT) for primary sclerosing cholangitis and developed invasive tissue disease due to $M$. capitatus. BDG was done weekly to monitor the antifungal therapy. However in the 5th week there was sudden rise in BDG $>523 \mathrm{pg} / \mathrm{ml}$ despite dual antifungal. This was used as a guide to investigate for further persistence of focus for IFI and source control. M. capitatus was intermediate resistant to voriconazole and echinocandins. Voriconazole was stopped and LAmB was continued for 6 month. In our experience, biomarker BDG is proved helpful for diagnosis, monitoring the therapeutic efficacy and used as guide to decide the duration of antifungal therapy. J Microbiol Infect Dis 2020; 10(2):98-102.
\end{abstract}

Keywords: Beta D Glucan, Antifungal, M. capitatus

\section{INTRODUCTION}

Invasive fungal infections (IFI) remains major cause of morbidity and mortality in liver transplant recipients (LTR) [1]. Most frequent causes of IFI are Candida and Aspergillus in these patients, however due to increasing use of antifungal prophylaxis and increasing antifungal resistance less common fungi are causing growing concern $[1,2]$. $M$. capitatus is an emerging opportunistic fungal infection, the majority $(91.7 \%)$ of these were reported in patients with hematological diseases $[3,4]$. $M$. capitatus is a teleomorph form of Spirochete capitata, previously named Geotrichum capitatum or Trichosporon capitatum or Blastoschizomyces capitatus, is a fungus, widespread in nature such as wood, soil and in animals [5]. It is part of the normal microbiota of human skin and mucosa of respiratory tracts and gastrointestinal system of healthy people [5]. One of the challenges in clinical medicine is the diagnosis of rare fungal infections, particularly in differentiating colonization from IFI in immunocompromised patients [6]. There is emerging evidence in literature, suggesting that the carbohydrate biomarker (1-3)- $\beta$-D-glucan may be useful, both in the diagnosis and therapeutic monitoring of IFI [6-9].

We describe first case report on use of BDG for monitoring antifungal therapy in a LTR with invasive tissue infection due to multifungal resistant $M$. capitatus.

\section{CASE REPORT}

A 59-year-old male was admitted to intensive care unit (ICU) post third liver transplant (LTX) for recurrent primary sclerosing cholangitis. Following the second LTX he had recurrent cholangitis episodes due to extended spectrum beta lactamase coliforms (ESBL E. coli) and treated with carbapenem. He was on prednisolone $5 \mathrm{mg} \mathrm{OD}$ and tacrolimus $0.5 \mathrm{mg}$ BD immunosuppression as per our protocol. The trough level was maintained $<10 \mu \mathrm{g} / \mathrm{ml}$.

On the first day of following third LTX in ICU he was requiring very high inotropic support, therefore he returned to theatre for a laparotomy and had wash out and mesh closure. He had sequential broad spectrum antibiotics

Correspondence: Dr. Anita Verma, Department of Infection Science and Institute of Liver Studies, King's College Hospital, Denmark Hill, London, SE5 9RS, United Kingdom

Email: anitaverma@nhs.net

Received: 31 January 2020 Accepted: 22 March 2020

Copyright (C JMID / Journal of Microbiology and Infectious Diseases 2020, All rights reserved 
piperacillin-tazobactam and meropenem and prophylactic antifungal (anidulafungin) as per unit protocol. As per our unit protocol serum BDG was measured weekly using Fungitell assay (Associates of Cape Cod, Inc.). Baseline BDG before transplant was below cut-off value $72 \mathrm{pg} / \mathrm{ml}$. On D7 relaparotomy and washout was done for bowel perforation.

Patient stayed on anidulafungin because of high fungal infection biomarker BDG and bowel perforation. On D14 he had another septic episode, while on meropenem, vancomycin and on anidulafungin. There was sudden rise of BDG to $>523 \mathrm{pg} / \mathrm{ml}$ (previously $297 \mathrm{pg} / \mathrm{ml}$ ) (Figure 1). On imaging, CT scan of thorax, abdomen and pelvis revealed right liver graft ischemic changes, subphrenic collections and right side pleural effusion. On day 22 he returned to theatre for further laparotomy and washout. There was necrotic omental tissue which was resected and right liver lobe was biopsied with diagnosis of acute cellular rejection. Omental tissue and abdominal fluid culture was positive for Enterococcus faecium, Candida albicans and $M$. capitatus. Blood cultures stayed negative. The diagnosis of $M$. capitatus was confirmed by matrix assisted laser desorption ionization-time of flight mass spectrometry (MALDI-TOF MS). Anidulafungin was changed to liposomal amphotericin B (LAmB) $5 \mathrm{mg} / \mathrm{kg}$ and voriconazole was added. Antifungal susceptibility confirmed $M$. capitatus isolate was resistant to echinocandins, fluconazole and intermediate resistant to voriconazole. Voriconazole was stopped after more than a week of treatment and continued with high dose LAmB and started on meropenem.

Initially BDG came down to normal but start rising D38 while on antifungals (Figure 1). He had interval CT imaging showing stable areas of infarction in the right lobe and right side pleural effusion. On D40 a wedge biopsy of a suspected abscess at the right lobe was taken. Culture and histology confirmed $M$. capitatus invasive fungal infection. Microscopic examination showed large abscessified hepatic infarcts and necrotizing granulomata, with abundant fungal organisms with images of angio invasive growth at the infarcted areas (Figure 2). Liver tissue grew $M$. capitatus in culture, and was only sensitive to Amphotericin B. We continued LAmB and meropenem was given for 4 weeks.

However on D118 he had another peak of very high BDG $>523 \mathrm{pg} / \mathrm{ml}$, while on high dose LAmB. Blood culture was negative. All false positive (e.g. albumin infusion, IVIG, surgical packing, dialysis, or tazocin) causes of BDG were ruled out. CT scans showed infarcted liver segment VII and VIII and sub-hepatic and subphrenic abscesses. He had laparotomy \& excision of infected liver tissue segment VII and VIII and drainage of collection.

Tissue histology remained positive for fungus but culture negative. The collection was positive for vancomycin resistant $E$. faecium (VRE), which was treated with sequential linezolid 600 $\mathrm{mg}$ PO BD and tigecycline $100 \mathrm{mg} \mathrm{BD} \mathrm{IV} \mathrm{for} 3$ weeks. Antifungal LAmB $5 \mathrm{mg} / \mathrm{kg}$ /day IV dose was continued till BDG was normal. There were no drug related side effects during this long term treatment with $\mathrm{LAmB}$.

After source control and 6 month of LAmB treatment his BDG levels came back to normal range (Figure 1). After $>1$ year follow up patient is well and repeat CT scan was normal with normal liver graft appearance and no intraabdominal collections.

\section{DISCUSSION}

The presentation of $M$. capitatus can be often nonspecific resembling other invasive fungal infections. $M$. capitatus is a poorly invasive organism unlike other Candida and mould infection. Mere isolation of the fungus from sputum or stool or drain or line site wound swabs does not establish the diagnosis. We demonstrated on culture and on histology hyphal forms and arthrospores in the liver tissue (Figure 1). Up to $60-80 \%$ patients develop deep tissue involvement, compared to $10-20 \%$, who present with blood stream infection [3]. In our case blood culture was always negative. The most common risk factor was profound neutropenia. The other risk factors are use of broad-spectrum antibiotics, corticosteroids, central catheter, cytotoxic chemotherapy, and the alteration of local defenses of the skin and mucosa by breakdown [4]. In our case the risk factors for invasive $M$. capitatus infection were bowel perforation and likely portal of entry, was open surgical wound.

The treatment of $M$. capitatus is limited because of its resistant antifungal profile [4]. It is intrinsically resistant to echinocandins, among the azoles it is more active towards voriconazole, posaconazole and itraconazole in vitro than fluconazole. Amphotericin B alone or in combination with flucytosine is the most effective regimen [4]. 
Table 1. MIC for Magnusiomyces capitatus compared to the CLSI Tables of clinical breakpoints for antifungal agents for C. albicans.

\begin{tabular}{|c|c|c|c|c|}
\hline \multirow{3}{*}{ Antifungal agent } & \multirow{2}{*}{\multicolumn{4}{|c|}{$\begin{array}{c}\text { CLSI antifungal breakpoints } \\
\text { C. albicans }\end{array}$}} \\
\hline & M. capitatus & & & \\
\hline & & $\mathrm{s}$ & 1 & $R=\geq$ \\
\hline Anidulafungin & $1(\mathrm{R})$ & $\leq 0.025$ & 0.5 & $\geq 1$ \\
\hline Caspofungin & $1(\mathrm{R})$ & $\leq 0.025$ & 0.5 & $\geq 1$ \\
\hline Micafungin & $1(\mathrm{R})$ & $\leq 0.025$ & 0.5 & $\geq 1$ \\
\hline Fluconazole & $8(\mathrm{R})$ & $\leq 2$ & 4 & $\geq 8$ \\
\hline Voriconazole & $0.5(\mathrm{I})$ & $\leq 0.12$ & $0.25-0.5$ & $\geq 1$ \\
\hline Amphotericin B & $1(\mathrm{~S})$ & $\leq 1$ & 0.5 & $\geq 1$ \\
\hline
\end{tabular}

CLSI: The Clinical and Laboratory Standards Institute, $S=$ sensitive, R=resistant

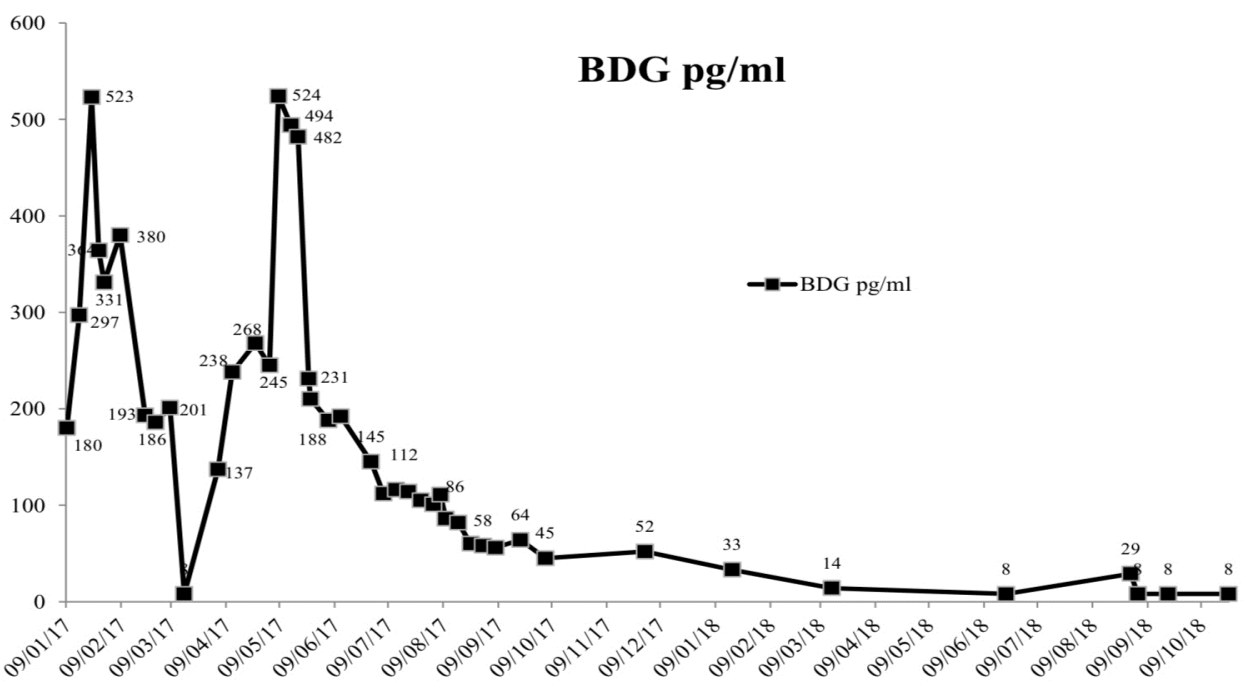

Figure 1. Pattern of Beta-D-Glucan (BDG) a fungal infection biomarker for monitoring antifungal treatment.

In our case, patient stayed on anidulafungin for two weeks, but after identification of yeast in culture, we started combination therapy with Liposomal amphotericin B (LAmB) and voriconazole. Despite on high dose (LAmB) antifungals BDG start rising which may be used indicator for failure of therapy due to resistance or likely persistence of focus.

In clinical practice the most challenging aspect for treating tissue IFI is monitoring the efficacy of treatment and duration of therapy. Emerging data suggest that, biomarker BDG is useful in both diagnosis and therapeutic monitoring of IFI due to some moulds, yeasts, and dimorphic fungi [7-10]. The BDG assay can be positive in patients with a variety of IFIs, including Pneumocystis jirovecii, aspergillosis and candidiasis but it is typically negative in patients with cryptococcosis, fusariosis and mucormycosis [7-10]. We suspected the diagnosis of IFI because of high fungal biomarker BDG and escalated investigations to identify focus. To our knowledge, no other study has reported the use of serum BDG for detection of $M$. capitatus. However, the detection of serum galactomannan (GM) in the diagnosis of $M$. capitatus has been reported in a disseminated infection [11]. In our case serum GM was not positive. A meta-analysis and a large multicenter 
study showed that BDG was able to distinguish proven or probable IFIs from no IFIs in several patient populations [10].

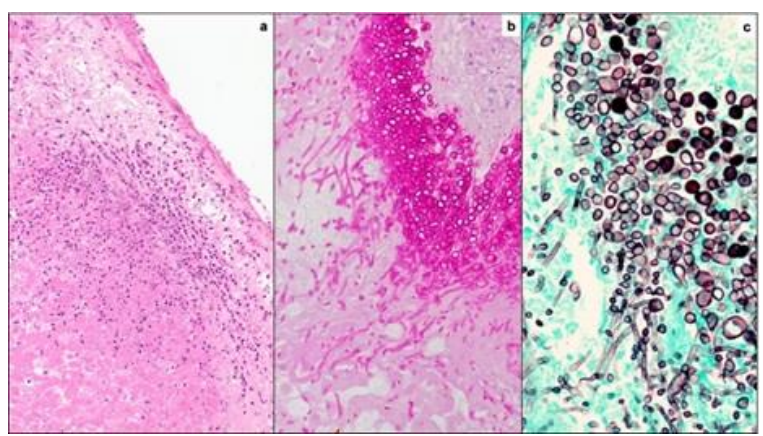

Figure 2. a) Resected wedge liver tissue with coagulative necrosis of the parenchyma in keeping with infarcted tissue associated with focal neutrophilic infiltrate (H\&E). b-c) Abundant fungal organisms were identified within the necrotic tissue, with spores and hyphal filamentous structures, DPAS (b) and Grocott (c) stains. Detail of the fungus on Grocott stain.

BDG serum result can be false positive if patient has concurrent bacteremia, hemodialysis membranes, administrations of blood products, albumin or immunoglobulin products. It is used as an adjunct in the diagnosis of IFI. It is proved useful in some cases for therapeutic monitoring [7] and most studies has consistently demonstrated the high negative predictive value which has been used to rule out the IFI.[9, 10]. Galactomannan another fungal infection biomarker was used to identify infection due Geotrichum capitatum in hematology patients but in our case it was negative [11].

In our experience, persistently high BDG can be used as guide for persistence of IFI and as guide for escalating the investigations for IFI and source control. In this case after source control and appropriate antifungal treatment the BDG came down to normal values Increasing BDG levels or repeatedly positive results may lead to early initiation or change of antifungal therapy. Conversely, decreasing BDG levels may lead to appropriate cessation of antifungal therapy, which may prevent treatment-associated complications as well as the emergence of fungal resistance.

In conclusion, we successfully treated the multiantifungal resistant invasive $M$. capitatus infection in a liver transplant recipient due to early BDG antigen detection and species identification with MALDI-TOF MS. The present case study illustrates the importance of speciation of the yeast and antifungal susceptibility testing. In our case IFI biomarker BDG proved very effective for monitoring the therapeutic efficacy and in deciding the duration of antifungal therapy. The BDG testing can play an important role in monitoring and diagnosis in selected patients with IFIs and further research will be crucial to properly implement this important assay in clinical practice.

\section{ACKNOWLEDGMENTS}

All authors contributed to the preparation of this article. There was no external funding for this project.

Declaration of Conflicting Interests: The authors declare that they have no conflict of interest.

Financial Disclosure: No financial support was received.

\section{REFERENCES}

1. Verma A, Weigel KS, Dexter SYK, Dhawan A. Evolution in the management of invasive fungal infections in liver transplant recipient. OBM Transplantation 2018; 2, doi:10.21926/obm.transplant.1802009

2. Pappas PG, Alexander BD, Andes DR, et al. Invasive fungal infections among organ transplant recipients: results of the Transplant-Associated Infection Surveillance Network (TRANSNET). Clin Infect Dis 2010; 50:1101-1111.

3. Girmenia C, Pagano L, Martino B, et al. Invasive infections caused by Trichosporon species and Geotrichum capitatum in patients with hematological malignancies: a retrospective multicenter study from Italy and review of the literature. J Clin Microbiol. 2005; 43 (4):18181828.

4. Mazzocato S, Marchionni E, Fothergill AW, et al. Epidemiology and outcome of systemic infections due to Saprochaete capitata: case report and review of the literature. Infection. 2014; 43: 211 215.

5. Döğen A, Kaplan E, Öksüz Z, Serin MS, Ikit M, de Hoog GS. Dishwashers are a major source of human opportunistic yeast-like fungi in indoor environments in Mersin, Turkey. Med Mycol 2013; 51:493-498.

6. Arendrup $\mathrm{MC}$, Boekhout $\mathrm{T}$, Akova $\mathrm{M}$, et al . ESCMID and ECMM joint clinical guidelines for the diagnosis and management of rare invasive yeast infections. European Society of Clinical Microbiology and Infectious Diseases Fungal Infection Study Group; European Confederation of 
Medical Mycology. Clin Microbiol Infect 2014; 20:76-98.

7. Pickering JW; Sant HW, Bowles CA, Roberts, WL, Woods GL. Evaluation of a $(1,3)-\beta-D$-glucan assay for diagnosis of invasive fungal infections. J. Clin. Microbiol 2005; 43:5957-5962.

8. Levesque E, Rizk F, Noorah Z, et al. Detection of $(1,3)-\beta-D-G l u c a n$ for the Diagnosis of Invasive Fungal Infection in Liver Transplant Recipients. Int J Mol Sci 2017; 18(4):862.

9. McCarthy MW, Petraitiene R, Walsh TJ. Translational Development and Application of $(1,3)-\beta-d-G l u c a n$ for Diagnosis and Therapeutic Monitoring of Invasive Mycoses. International Journal of Molecular Sciences 2017; 18(6), doi: 10.3390/ijms18061124.

10. He S, Hang JP, Zhang L, Wang F, Zhang DC, Gong $\mathrm{FH}$. A systematic review and meta-analysis of diagnostic accuracy of serum 1,3-beta-D-glucan for invasive fungal infection: Focus on cutoff levels. J Microbiol Immunol Infect 2015; 48(4):351361.

11. Özkaya-Parlakay A, Cengiz AB, Karadağ-Öncel E, et al. Geotrichum capitatum septicemia in a hematological malignancy patient with positive galactomannan antigen: case report and review of the literature. 